\title{
COMPOSIÇÃO QUÍMICA DOS ÓLEOS ESSENCIAIS DAS FOLHAS DE SEIS ESPÉCIES DO GÊNERO Baccharis DE “CAMPOS DE ALTITUDE” DA MATA ATLÂNTICA PAULISTA
}

\author{
João Henrique G. Lago*, Paulete Romoff e Oriana A. Fávero
}

Centro de Ciências e Humanidades, Universidade Presbiteriana Mackenzie, 01302-907 São Paulo - SP, Brasil

Marisi G. Soares, Patrícia T. Baraldi e Arlene G. Corrêa

Departamento de Química, Universidade Federal de São Carlos, 13565-905 São Carlos - SP, Brasil

Fátima O. Souza

Seção de Curadoria do Herbário, Instituto de Botânica, 04301-902 São Paulo - SP, Brasil

Recebido em 20/3/07; aceito em 20/9/07; publicado na web em 10/3/08

\begin{abstract}
COMPOSITION OF ESSENTIAL OILS FROM THE LEAVES OF SIX SPECIES OF THE Baccharis GENUS FROM "CAMPOS DE ALTITUDE" OF THE ATLANTIC FOREST OF SÃO PAULO. The essential oils from the leaves of six species of the Baccharis genus (B. dracunculifolia, B. microdonta, B. regnelli, B. schultzii, B. trimera, and B. uncinella), collected in the "Campos de Altitude" of the Atlantic Forest (SP), were extracted using hydrodistillation procedures and analyzed by GC and GC/MS. There was a predominance of sesquiterpenes in all studied oils as $\beta$-elemene in $B$. dracunculifolia and B. regnelli, $\alpha$-humulene in B. trimera, $\gamma$ gurjunene in $B$. schultzii, bicyclogermacrene in B. regnelli, $\delta$-cadinene in B. regnelli and B. uncinella, spathulenol in $B$. schultzii, caryophyllene oxide in $B$. microdonta and guaiol in $B$. uncinella. However, a high amount of monoterpenes was also observed in B. uncinella ( $\alpha$-pinene), B. regnelli ( $\delta$-car-3-ene) and B. schultzii (limonene). The chemical compounds of the essential oils of B. schultzii, B. regnelli and B. microdonta are described for the first time in this work.
\end{abstract}

Keywords: Asteraceae; Baccharis ssp.; essential oil composition.

\section{INTRODUÇÃO}

O gênero Baccharis L. (Asteraceae), caracterizado botanicamente por apresentar espécimes na forma de arbustos ou até mesmo como árvores pequenas, conta com aproximadamente 400 espécies distribuídas predominantemente na América do Sul, sendo 120 delas de ocorrência brasileira. ${ }^{1}$ Tais espécies são encontradas principalmente no sul e sudeste do país, principalmente nas regiões de Campos de Altitude que consistem em biomas que compreendem uma série de formações fresco-úmidas, dominadas por gramíneas e arbustos, restritas às regiões mais elevadas (que começam em alturas de 1800-2000 m) da região da Mata Atlântica. ${ }^{2}$

Diversas espécies de Baccharis são usadas na medicina popular para tratamento de dor de cabeça, diabetes e desordens hepáticas, principalmente devido ao acúmulo de metabólitos secundários bioativos, tais como terpenóides, flavonóides, cromenos, etc. ${ }^{3-10}$ A ocorrência de diversos terpenóides, principalmente monoterpenos e sesquiterpenos, foi também observada nos óleos essenciais de Baccharis articulata, ${ }^{11,12}$ B. caprariaefolia, ${ }^{13}$ B. cognata, ${ }^{11}$ B. crispa, ${ }^{12}$ B. coridifolia,${ }^{14}$ B. dracunculifolia,${ }^{13,15-17}$ B. erioclada, ${ }^{13}$ B. halimifolia, ${ }^{18} \mathrm{~B}$. latifolia,${ }^{15} \mathrm{~B}$. linearis,${ }^{19} \mathrm{~B}$. microcephala, ${ }^{12}$ B. milleflora, ${ }^{11,12}$ B. myriocephala, ${ }^{12}$ B. obovata, ${ }^{20}$ B. oxyodonta ${ }^{11}$ B. platipoda,$^{13}$ B. racemosa ${ }^{19}$ B. salicilifolia, ${ }^{15,20,21}$ B. semiserrata,${ }^{11}$ B. spartioides, ${ }^{22}$ B. stenocephala,$^{12}$ B. tenella ${ }^{23}$ B. tricuneata, ${ }^{24,25}$ B. tridentata, ${ }^{13}$ B. trimera, ${ }^{12}$ B. trinervis,${ }^{26}$ B. uncinella,${ }^{11}$ B. usterii ${ }^{12}$ e de $B$. vincaefolia. ${ }^{13}$

Uma recente revisão descreve a influência de diferentes fatores, tais como parâmetros microclimáticos, altitude, sazonalidade, desenvolvimento, nutrientes e/ou disponibilidade hídrica na produção e/ou acúmulo de metabólitos secundários, tais como óleos essenciais..$^{27}$ Desta forma, dada as diferenças nos parâmetros encon-

*e-mail: joaolago@mackenzie.com.br trados nas regiões de Campos de Altitude, o objetivo deste trabalho foi descrever a composição química dos óleos essenciais das folhas de seis espécies de Baccharis coletadas na cidade de Campos do Jordão, estado de São Paulo. As espécies estudadas foram $B$. dracunculifolia DC., B. trimera (Less) DC., B. uncinella DC., B. schultzii Baker, B. regnelli Sch. Bip. e B. microdonta DC., sendo a composição dos óleos essenciais das três ultimas espécies descrita pela primeira vez neste trabalho.

\section{RESULTADOS E DISCUSSÃO}

Os rendimentos dos óleos essenciais obtidos das folhas de $B$. dracunculifolia, B. microdonta, B. regnelli, B. uncinella, B. schultzii e B. trimera variaram entre $0,08-0,21 \%$. Foram identificados 67 compostos (que correspondem a 70-92\% do conteúdo total dos óleos), como mostrado na Tabela 1.

Comparativamente, a composição química dos óleos essenciais dessas seis espécies de Baccharis revelou uma proporção elevada de monoterpenos em B. schultzii (34,93\%), em B. regnelli $(35,79 \%)$ e em $B$. uncinella $(21,34 \%)$, enquanto que esses compostos são minoritários apenas em $B$. dracunculifolia $(0,30 \%)$. As quantidades de monoterpenos nos óleos de $B$. microdonta e de $B$. trimera mostraram-se similares, com proporções de 9,23 e 7,20\%, respectivamente. Por outro lado, os hidrocarbonetos sesquiterpênicos foram mais abundantes em $B$. dracunculifolia $(63,10 \%)$, B. regnelli $(37,76 \%)$ e $B$. trimera $(53,52 \%)$, enquanto que no óleo de $B$. microdonta os sesquiterpenos oxigenados foram predominantes $(49,91 \%)$. Em B. schultzii e em B. uncinella, as proporções relativas de hidrocarbonetos sesquiterpênicos e de sesquiterpenos oxigenados mostraram-se similares $(29,33 / 28,30 \%$ e $26,33 / 22,28 \%$, respectivamente).

O óleo de B. dracunculifolia mostrou-se constituído por 17 componentes, dos quais somente $0,30 \%$ correspondem a monoterpenos 
Tabela 1. Percentual relativo dos componentes do óleo essencial das folhas de seis espécies do gênero Baccharis

\begin{tabular}{|c|c|c|c|c|c|c|c|}
\hline \multirow[t]{2}{*}{ Composto } & \multirow[t]{2}{*}{ IK } & \multicolumn{6}{|c|}{ Espécies do gênero Baccharis } \\
\hline & & $B d$ & $B m$ & $\mathrm{Br}$ & Bs & $B t$ & $B u$ \\
\hline 1. $\alpha$-Pineno & 939 & - & 8,11 & 1,14 & 1,03 & 1,11 & 13,59 \\
\hline 2. $\alpha$-Fencheno & 951 & - & - & - & - & - & 0,27 \\
\hline 3. Benzaldeído & 961 & 0,05 & - & - & - & - & - \\
\hline 4. Sabineno & 976 & - & - & 2,26 & - & - & 0,29 \\
\hline 5. $\beta$-Pineno & 980 & 0,05 & 1,12 & - & 0,37 & - & 6,69 \\
\hline 6. Trans-m-menta-2,8-dieno & 981 & - & - & - & - & - & 0,42 \\
\hline 7. Mirceno & 991 & - & - & 2,42 & - & 6,09 & - \\
\hline 8. $\delta$-Car-2-eno & 1001 & - & - & 1,06 & - & - & - \\
\hline 9. Isosilvestreno & 1007 & - & - & 2,80 & - & - & - \\
\hline 10. $\delta$-Car-3-eno & 1011 & - & - & 15,42 & - & - & - \\
\hline 11. $\beta$-Felandreno & 1031 & - & - & 0,44 & - & - & - \\
\hline 12. Limoneno & 1032 & - & - & - & 33,50 & - & - \\
\hline 13 Z,ß-Ocimeno & 1040 & - & - & 3,20 & - & - & - \\
\hline $14 E, \beta$-Ocimeno & 1050 & - & - & 0,32 & - & - & - \\
\hline 15. Hidrato de $c i s$-sabineno & 1068 & - & - & 0,18 & - & - & 0,08 \\
\hline 16. Canfenilona & 1083 & 0,04 & - & - & - & - & - \\
\hline 17. Mircenol & 1118 & - & - & 0,68 & - & - & - \\
\hline 18.Cânfora & 1143 & - & - & 0,44 & - & - & - \\
\hline 19. $\alpha$-Terpineol & 1189 & 0,21 & - & 0,52 & - & - & - \\
\hline 20. Geraniol & 1255 & - & - & 1,80 & - & - & - \\
\hline 21. $p$-Ment-1-en-9-ol & 1291 & - & - & - & 0,03 & - & - \\
\hline 22. Acetato de $c i s$-pinocarvila & 1309 & - & - & 3,11 & - & - & - \\
\hline 23. 2-Fenil propanoato de etila & 1350 & 7,83 & - & - & - & - & - \\
\hline 24. Isoledeno & 1373 & - & - & - & - & 1,02 & - \\
\hline 25. Damascenona & 1380 & - & - & - & - & 0,24 & - \\
\hline 26. $\beta$-Cubebeno & 1390 & - & - & - & 1,76 & - & - \\
\hline 27. $\beta$-Elemeno & 1391 & 53,31 & - & 10,84 & - & 1,68 & 3,11 \\
\hline 28. $\beta$-Longipineno & 1398 & - & - & - & - & 0,11 & - \\
\hline 29. Z-Cariofileno & 1404 & - & - & - & - & 2,01 & - \\
\hline 30. E-Cariofileno & 1418 & 1,96 & 0,04 & - & 8,34 & - & 3,58 \\
\hline 31. $\beta$-Gurjuneno & 1432 & - & - & - & - & 1,29 & - \\
\hline 32. $\gamma$-Elemeno & 1433 & - & 0,39 & 1,40 & - & - & - \\
\hline 33. Aromadendreno & 1439 & 0,58 & 0,52 & - & - & 0,43 & 0,27 \\
\hline 34. $\alpha$-Patchouleno & 1454 & - & - & - & - & 2,88 & - \\
\hline 35. $\alpha$-Humuleno & 1456 & - & 0,30 & - & - & 19,44 & 1,46 \\
\hline 36. Allo-aromadendreno & 1461 & - & - & - & 0,32 & - & - \\
\hline 37. $\beta$-Santaleno & 1462 & 0,64 & - & - & - & - & - \\
\hline 38. $\gamma$-Gurjuneno & 1473 & - & - & - & 13,24 & - & - \\
\hline 39. $\gamma$-Muuroleno & 1477 & - & 4,68 & - & - & - & - \\
\hline 40. Germacreno D & 1480 & 3,99 & - & - & - & 8,86 & 3,28 \\
\hline 41. Biciclogermacreno & 1494 & 1,02 & 0,72 & 11,58 & 2,99 & 2,74 & - \\
\hline 42. $\alpha$-Muuroleno & 1499 & - & 4,58 & - & - & - & - \\
\hline 43. Germacreno A & 1503 & - & - & - & - & 7,80 & 1,39 \\
\hline 44. $\delta$-Cadineno & 1524 & - & 2,37 & 12,82 & 2,68 & 0,57 & 13,24 \\
\hline 45. Cadina-1,4-dieno & 1532 & 1,60 & - & - & - & 0,88 & - \\
\hline 46. $\alpha$-Calacoreno & 1542 & - & - & - & - & 0,18 & - \\
\hline 47. Germacreno-B & 1556 & - & 3,19 & 1,12 & - & 3,39 & - \\
\hline 48. Butirato de Z-geranila & 1562 & - & - & 0,46 & - & - & - \\
\hline 49. E-Nerolidol & 1564 & 2,30 & - & 3,62 & - & - & - \\
\hline 50. Espatulenol & 1576 & - & 0,79 & - & 17,40 & - & - \\
\hline 51. Óxido de cariofileno & 1581 & - & 24,06 & - & - & - & - \\
\hline 52. Tujopsan-2 $\alpha-\mathrm{ol}$ & 1582 & - & - & - & - & 5,94 & - \\
\hline 53. Globulol & 1583 & - & - & - & - & 1,66 & - \\
\hline 54. Viridiflorol & 1590 & - & 7,67 & - & - & - & - \\
\hline 55. Guaiol & 1595 & - & - & - & - & - & 11,35 \\
\hline 56. Epi- $\alpha$-cadinol & 1640 & - & - & - & 6,31 & - & - \\
\hline 57. Epi- $\alpha$-muurolol & 1641 & - & 5,76 & 2,38 & - & 0,83 & - \\
\hline 58. Cubenol & 1642 & - & - & - & - & 5,58 & 8,25 \\
\hline 59. $\alpha$-Cadinol & 1653 & - & 8,44 & - & 1,56 & 0,18 & - \\
\hline
\end{tabular}


Tabela 1. continuação

\begin{tabular}{|c|c|c|c|c|c|c|c|}
\hline \multirow[t]{2}{*}{ Composto } & \multirow[t]{2}{*}{ IK } & \multicolumn{6}{|c|}{ Espécies do gênero Baccharis } \\
\hline & & $B d$ & $B m$ & $B r$ & Bs & $B t$ & $B u$ \\
\hline 60. 14-hidroxi-9-epi-E-cariofileno & 1664 & 3,62 & - & - & - & - & - \\
\hline 61. Kusinol & 1674 & - & - & - & - & 0,36 & - \\
\hline 62. Acetato de $Z$-nerolidila & 1675 & - & 3,19 & 0,12 & - & - & - \\
\hline 63. Cis-14-Muurol-5-en-4-ona & 1682 & - & - & - & - & - & 2,68 \\
\hline 64. Acorenona & 1685 & - & - & - & 3,03 & - & - \\
\hline 65. Z,Z-Farnesol & 1713 & 1,72 & - & - & - & - & - \\
\hline 66. E,E-Farnesol & 1722 & 1,23 & - & - & - & - & - \\
\hline 67. Hexadecanoato de etila & 1993 & 0,38 & - & - & - & - & - \\
\hline Hidrocarbonetos monoterpênicos & & 0,09 & 9,23 & 29,24 & 34,90 & 7,20 & 21,34 \\
\hline Monoterpenos oxigenados & & 0,21 & - & 6,55 & 0,03 & - & - \\
\hline Hidrocarbonetos sesquiterpênicos & & 63,10 & 16,79 & 37,76 & 29,33 & 53,52 & 26,33 \\
\hline Sesquiterpenos oxigenados & & 8,87 & 49,91 & 6,58 & 28,30 & 14,55 & 22,28 \\
\hline Benzenóides & & 7,88 & - & - & - & - & - \\
\hline Derivado de ácido graxo & & 0,38 & - & - & - & - & - \\
\hline TOTAL & & 80,53 & 75,93 & 80,13 & 92,56 & 75,27 & 69,95 \\
\hline
\end{tabular}

$\mathrm{Bd}=$ B. dracunculifolia $; \mathrm{Bm}=B$. microdonta $; \mathrm{Br}=B$. regnelli; $\mathrm{Bs}=B$. schultzii $; \mathrm{Bt}=B$. trimera $; \mathrm{Bu}=B$. uncinel

( $\beta$-pineno, canfenilona e $\alpha$-terpineol), enquanto que os sesquiterpenos são os constituintes principais $(71,97 \%)$, sendo $63,10 \%$ correspondentes aos hidrocarbonetos e $8,87 \%$ aos derivados oxigenados (Tabela 1). O sesquiterpeno não oxigenado $\beta$-elemeno foi identificado como o componente majoritário $(53,31 \%)$ através de experimento de $\mathrm{CG} / \mathrm{EM}$ do óleo bruto em associação à análise por RMN de ${ }^{1} \mathrm{H}$ e ${ }^{13} \mathrm{C}$ da mistura ${ }^{28}$ seguido da comparação com dados da literatura. ${ }^{29}$ Em trabalho anterior, ${ }^{13}$ o óleo de $B$. dracunculifolia, cujas folhas foram coletadas na região de Campinas/SP, mostrou uma baixa concentração de monoterpenos $(6,27 \%)$ e uma quantidade elevada de sesquiterpenos $(51,1 \%)$, sendo os derivados não oxigenados os principais $(26,8 \%)$. Em outro trabalho,,$^{15}$ o óleo das partes aéreas de um espécime boliviano de altitude mostrou uma maior proporção de monoterpenos $(29,1 \%)$, porém sendo composto majoritariamente de sesquiterpenos $(64,2 \%)$, dos quais $48,0 \%$ são derivados não-oxigenados. No entanto, embora os resultados qualitativos sejam diferentes, observa-se uma predominância dos derivativos sesquiterpênicos não oxigenados nos óleos de todas as espécies estudadas.

O óleo de B. dracunculifolia ("óleo de vassoura") é empregado na indústria de perfumaria devido a seu aroma exótico, devido principalmente aos constituintes da fração oxigenada, mais especificamente $(E)$ nerolidol e espatulenol ${ }^{17}$. No presente trabalho, a quantidade relativa de $(E)$-nerolidol foi de 2,30\% não sendo detectado espatulenol, sugerindo que este óleo não está apropriado para o uso comercial.

Embora tenha sido observada a predominância dos hidrocarbonetos sesquiterpênicos como derivados principais em $B$. dracunculifolia, no óleo essencial de $B$. microdonta foi detectada uma concentração elevada dos sesquiterpenos oxigenados $(49,91 \%)$, principalmente óxido de cariofileno $(24,06 \%), \quad \alpha$-cadinol $(8,44 \%)$ e viridiflorol $(7,67 \%)$ além de uma maior proporção de monoterpenos $(9,23 \%)$, sendo $\alpha-$ e $\beta$ pinenos os principais $(8,11$ e $1,12 \%$, respectivamente).

O óleo de B. uncinella mostrou-se constituído por 13,59\% e $6,69 \%$ de $\alpha$ - e $\beta$-pinenos, respectivamente (Tabela 1), similar ao observado para o óleo essencial desta espécie, cujas folhas foram coletadas do sul do Brasil. ${ }^{11}$ No presente trabalho, a quantidade relativa de hidrocarbonetos sesquiterpênicos foi de aproximadamente $26 \%$, sendo $\delta$-cadineno o componente principal $(13,24 \%)$, enquanto que os constituintes mais abundantes da fração oxigenada $(22,28 \%)$ foram guaiol $(11,35 \%)$ e cubenol $(8,25 \%)$. Embora os sesquiterpenos oxigenados ocorram em uma proporção similar em ambos os óleos, foi observado um perfil qualitativo diferente daquele analisado anteriormente, ${ }^{11} \mathrm{o}$ qual mostrou ser constituído por aproximadamente 47,70\% de espatulenol, não detectado na análise atual (Tabela 1).

O óleo bruto de B. schultzii mostrou-se composto principalmente por monoterpenos $(34,93 \%)$, sendo limoneno $(33,50 \%)$ o constituinte principal. No entanto, apesar do alto teor de monoterpenos, os sesquiterpenos são ainda os componentes majoritários $(57,53 \%)$ destacando-se os derivados $\gamma$-gurjuneno $(13,24 \%)$ e espatulenol $(17,40 \%)$.

De modo similar aos óleos de B. schultzii e B. uncinella, o óleo de $B$. regnelli mostrou uma proporção elevada de monoterpenos $(35,79 \%)$, sendo o $\delta$-car-3-eno o constituinte mais abundante $(15,42 \%)$. Como observado na Tabela $1,44,34 \%$ do óleo bruto corresponde a hidrocarbonetos sesquiterpênicos $(37,76 \%)$ sendo biciclogermacreno $(11,58 \%), \delta$-cadineno $(12,82 \%)$ e $\beta$-elemeno $(10,84 \%)$ os componentes principais.

A diferenciação estrutural dos metabólitos voláteis detectados nas espécies de Baccharis estudadas mostrou-se significativa, visto que, dos 67 compostos identificados, apenas seis foram detectados simultaneamente em quatro ou cinco espécies. Dentre esses, o $\alpha$ pineno (B. microdonta, B. regnelli, B. schultzii, B. trimera e $B$. uncinella), o $\beta$-pineno ( $B$. dracunculifolia, B. microdonta, $B$. schultzii e $B$. uncinella), o E-cariofileno ( $B$. dracunculifolia, $B$. microdonta, B. schultzii e $B$. uncinella), o aromadendreno ( $B$. dracunculifolia, B. microdonta, B. trimera e B. uncinella), o biciclogermacreno (B. dracunculifolia, B. microdonta, $B$. regnelli, B. schultzii e B. trimera) e o $\delta$-cadineno (B. microdonta, B. regnelli, B. schultzii e B. uncinella).

Consequientemente, com base nos resultados obtidos no presente estudo (Tabela 1), observa-se uma tendência no acúmulo de sesquiterpenos como componentes principais dos óleos essenciais das folhas de espécies do gênero Baccharis coletadas em região de "Campos de Altitude".

\section{PARTE EXPERIMENTAL}

\section{Material vegetal}

As folhas de Baccharis dracunculifolia (PMSP8761), B. microdonta (PMSP8980), B. regnelli (PMSP8759), B. schultzii 
(SP382049), B. trimera (PMSP8758) e B. uncinella (SP382050) foram coletadas em região de "Campos de Altitude" na cidade de Campos do Jordão, SP, em junho de 2005 (período da manhã). Os espécimes estudados foram identificados pela Profa. F. O. Souza (Instituto de Botânica - SP) e as exsicatas depositadas no Herbário da Prefeitura Municipal de São Paulo (PMSP) e no Herbário de Instituto de Botânica (SP).

\section{Extração dos óleos essenciais}

As folhas frescas das seis espécies foram submetidas, separadamente, a procedimento de hidrodestilação em um aparelho de Clevenger durante $4 \mathrm{~h}$. Após a extração com $\mathrm{CH}_{2} \mathrm{Cl}_{2}$, os óleos essenciais foram secados sobre $\mathrm{Na}_{2} \mathrm{SO}_{4}$ anidro e filtrados. Após evaporação do solvente e pesagem, o rendimento de cada óleo essencial foi calculado tendo como base a massa de óleo essencial e de folhas frescas: Baccharis dracunculifolia $(0,21 \%)$, B. microdonta $(0,09 \%)$, $B$. regnelli $(0,12 \%)$, B. schultzii $(0,08 \%)$, B. trimera $(0,15 \%)$ e $B$. uncinella $(0,17 \%)$. As análises químicas dos óleos brutos foram executadas imediatamente após obtenção dos mesmos.

\section{Análise dos óleos essenciais}

\section{Análise por $C G$ e $C G / E M$}

A análise por CG foi executada em um cromatógrafo HewlettPackard 5890 - séries II (gás de arraste: He) equipado com detector FID, coluna capilar HP-5 (5\% de difenil em $95 \%$ de dimetilpolissiloxano - comprimento: $30 \mathrm{~m}$, diâmetro interno: 0,32 mm, espessura do filme: 0,25 m), injetor automático HP 7673 (modo "split" 1:20) e integrador eletrônico HP 3396A. Programação de temperatura do forno: $60-280{ }^{\circ} \mathrm{C}$ com taxa de aquecimento de $3{ }^{\circ} \mathrm{C} / \mathrm{min}$ mantendose $10 \mathrm{~min}$ a $280{ }^{\circ} \mathrm{C}$. As temperaturas do injetor e do detector foram mantidas em 220 e $280{ }^{\circ} \mathrm{C}$, respectivamente. As concentrações dos componentes foram calculadas tendo como base as áreas dos picos no cromatograma, seguindo a ordem de eluição da coluna HP-5 (Tabela 1). A análise por CG/EM foi realizada em cromatógrafo HewlettPackard HP-6890 (coluna HP-5 - 5\% de difenil em 95\% de dimetilpolissiloxano - comprimento: $30 \mathrm{~m}$, diâmetro interno: $0,32 \mathrm{~mm}$, espessura do filme: 0,25 m, gás de arraste: He) acoplado a espectrômetro Hewlett-Packard HP-5973 (operando por impacto eletrônico - $70 \mathrm{ev}$ ), nas mesmas condições de programações de temperatura descritas acima.

\section{Análise por $\mathrm{RMN}$ de ${ }^{1} \mathrm{H} e{ }^{13} \mathrm{C}$}

Os espectros de RMN de ${ }^{1} \mathrm{H}$ e de ${ }^{13} \mathrm{C}$ NMR foram registrados em 500 e $125 \mathrm{MHz}$, respectivamente, em um espectrômetro Bruker modelo DRX-500 com a amostra dissolvida em $\mathrm{CDCl}_{3}$ com $1 \%$ de TMS (Aldrich).

\section{Identificação dos constituintes}

A identificação dos componentes foi baseada na comparação de seus espectros de massas com aqueles da base de dados do espectrômetro (Bibliotecas Wiley e NIST) e confirmada pela determinação de seus índices de Kovats, que foram calculados relativamente aos tempos da retenção de uma série de n-alkanos. ${ }^{30}$

\section{AGRADECIMENTOS}

À FAPESP e ao MackPesquisa pelo apoio financeiro. J. H. G. Lago e A. G. Correa são gratos ao CNPq pelas bolsas de produtividade a pesquisa.

\section{REFERÊNCIAS}

1. Giuliano, D. A.; Darwiniana 2001, 39, 131

2. Safford, H. D.; J. Biogeography 1999, 26, 693.

3. He, K.; Montenegro, G.; Hoffmann, J. J.; Timmermann B. N.; Phytochemistry 1996, 41, 1123.

4. Gené, R. M.; Cartaná, C.; Adzet, T.; Marín, E.; Parella, T.; Canigueral, S.; Planta Med. 1996 62, 232.

5. Jarvis, B. B.; Mokhtari-Rejali, N.; Schenkel, E. P.; Barros, C. S.; Matzenbacher, N. I.; Phytochemistry 1991, 30, 789.

6. Bohlmann, F.; Knauf, W.; King, R. M.; Robinson, H.; Phytochemistry 1979, 18, 1011 .

7. Labbe, C.; Rovirosa, J.; Faini, F.; Mahu, M.; San-Martin, A.; Castillo, M.; J. Nat. Prod. 1986, 49, 517.

8. Zdero, C.; Bohlmann, F.; Solomon, J. C.; King, R. M.; Robinson, H.; Phytochemistry 1988, 28, 531.

9. Queiroga, C. L.; Ferracini, V. L.; Marsaioli, A. J.; Phytochemistry 1996, 42, 1097.

10. Verdi, L. G.; Brighente, I. M. C.; Pizzolati, M. G.; Quim. Nova 2005, 28, 85.

11. Agostini, F.; Santos, A. C. A.; Rossato, M.; Pansera, M. R.; Zattera, F.; Wasum, R.; Serafini, L. A.; Rev. Bras. Farmacog. 2005, 15, 215.

12. Simões-Pires, C. A.; Debenedetti, S.; Spegazzini, E.; Mentz, L. A.; Matzenbacher, N. I.; Limberger, R. P.; Henriques, A. T.; Plant Syst. Evol. 2005, 253, 23.

13. Ferracini, V. L.; Paraiba, L. C.; Leitão-Filho, H. F.; Silva, A. G.; Nascimento, L. R.; Marsaioli, A. J.; J. Essent. Oil Res. 1995, 7, 355.

14. Dellacasa, A. D.; Bailac, P. N.; Ponzi, M. I.; Ruffinengo, S. R.; Eguaras, M. J.; J. Essent. Oil Res. 2003, 15, 282.

15. Loayza, I.; Abujder, D.; Aranda, R.; Jakupovic, J.; Collin, G.; Deslauriers, H.; Jean, F. I.; Phytochemistry 1995, 38, 381.

16. Queiroga, C. L.; Fukai, A.; Marsaioli, A. J.; J. Braz. Chem. Soc. 1990, 1, 105.

17. Cassel, E.; Frizzo, C. D.; Vanderlinde, R.; Atti-Serafini, L.; Lorenzo, D.; Dellacassa, E.; Ind. Eng. Chem. Res. 2000, 39, 4803.

18. Pino, J. A.; Marbot, R.; Payo, A.; Chao, D.; Herrera, P.; J. Essent. Oil Res. 2006, 18, 266.

19. Malizia, R. A. ; Cardell, D. A.; Molli, J. S.; González, S.; Guerra, P. E.; Grau, R. J.; J. Essent. Oil Res. 2005, 17, 103.

20. Malizia, R. A. ; Cardell, D. A.; Molli, J. S.; González, S.; Guerra, P. E.; Grau, R. J.; J. Essent. Oil Res. 2005, 17, 194.

21. Garcia, M.; Donadel, O. J.; Ardanaz, C. E.; Tonn, C. E.; Sosa, M. E.; Pest Manag. Sci. 2005, 61, 612.

22. van Baren, C. M.; Lira, P. D. L.; Bandoni, A. L.; Fortunato, R.; Mizrahi, I.; Juarez, M.; J. Essent. Oil Res. 2002, 14, 183.

23. Biurrun, F.; Juliani, R. H.; Lopez, M. L.; Zygadlo, J. A.; J. Essent. Oil Res. 2005, 17, 122

24. Arze, J. B. L. ; Garneau, F. X. ; Collin, G. ; Jean, F. I. ; Gagnon, H. ; J. Essent. Oil Res. 2004, 16, 429.

25. Arze, J. B. L.; Garneau, F. X.; Collin, G. ; Jean, F.I. ; Gagnon, H. ; J. Essent. Oil Res. 2004, 16, 429.

26. Albuquerque, M. R. J. R.; Souza, E. B.; Lins, M. U. D. S.; Nogueira, N. A P.; Lemos, T. L. G.; Silveira, E. R.; Pessoa, O. D. L.; Arkivoc 2004, VI, 59.

27. Gobbo-Neto, L.; Lopes, N. P.; Quim. Nova 2007, 30, 374.

28. Brochini, C. B.; Nuñez, C. V.; Moreira, I. C.; Roque, N. F.; Chaves, M. H.; Martins, D.; Quim. Nova 1999, 22, 37.

29. Adio, A. M.; Paul, C.; Kloth, P.; König, W. A.; Phytochemistry 2004, 65, 199.

30. Adams, R. P.; Identification of essential oil components by gas chromatography/quadrupole mass spectrometry, Academic Press: San Diego, 1995. 